\title{
High-conductivity graphene nanocomposite via facile, covalent linkage of gold nanoparticles to graphene oxide
}

\author{
ZHANG YuJie $^{1 \dagger}$, GENG MaKe $^{1 \dagger}$, ZHANG Huan $^{1}$, HE Yao $^{2}$, PENG Cheng $^{1}$, HUANG Qing $^{1 *} \&$ \\ FAN ChunHai ${ }^{1 *}$
}

${ }^{1}$ Laboratory of Physical Biology, Shanghai Institute of Applied Physics, Chinese Academy of Sciences, Shanghai 201800, China;

${ }^{2}$ Functional Nano \& Soft Materials Laboratory, Soochow University, Suzhou 215123, China

Received October 10, 2011; accepted April 6, 2012

\begin{abstract}
Graphene and its derivative, graphene oxide (GO) have been substantively used as the main framework for dispersing or building nanoarchitectures because of their excellent properties in electronics and catalysis. The requirement to obtain superior graphene-metal hybrid nanomaterials has led us to explore a facile way to design 4-aminobenzenethiol/1-hexanethiolate-protected gold nanoparticles (aAuNPs)-functionalized graphene oxide composite (aAuNPs-GO) in solution. We demonstrate that when aAuNPs with amino groups are exposed to GO, well-dispersed coverage of Au nanoparticles are mainly observed on the edge of GO sheet. In contrast, when 1-hexanethiolate-protected gold nanoparticles (hAuNPs) without amino groups are exposed to GO, hAuNPs simply aggregate on the surface of GO. This indicates that amino groups located on the surface of Au nanoparticles are an essential prerequisite for attachment of nearly monodispersed aAuNPs. The strategy described here for the fabrication of aAuNPs-GO provides a straightforward approach to develop graphene-based nanocomposites with undamaged sheets structure and good solubility and also improve the conductivity of GO sheets evidently.
\end{abstract}

graphene oxide, gold nanoparticles, nanocomposite, Michael addition reaction

Citation: Zhang Y J, Geng M K, Zhang H, et al. High-conductivity graphene nanocomposite via facile, covalent linkage of gold nanoparticles to graphene oxide. Chin Sci Bull, 2012, 57: 3086-3092, doi: 10.1007/s11434-012-5333-6

The two-dimensional graphene was the last carbon allotrope discovered in 2004 [1]. Graphene, a single layer of carbon atoms packed in a honeycomb $2 \mathrm{D}$ structure, is a fundamental building block for OD fullerenes, 1D nanotubes and 3D graphite [2,3]. It is extremely promising for graphene to become nanoscale building blocks of new nanocomposites because of its unusual nanostructure and extraordinary electronic properties [4-7]. One possible way to make use of these properties for applications would be to introduce metal nanoparticles onto graphene sheets [8,9]. These new carbon nanocomposites could not only show unique performance of metal nanoparticles [10] and grapheme [2,11-13], but also potentially show extra novel properties due to the

\footnotetext{
$\dagger$ These authors contributed equally to this work.

* Corresponding authors (email: huangqing@ @inap.ac.cn; fchh@sinap.ac.cn)
}

synergistic effect of metal nanoparticles and the thin atomic carbon layer [8,14-17]. Interestingly, metal-fullerene or carbon nanotubes (CNT) composites have been studied for several years due to their fascinating properties. For example, Au nanoparticles have been successfully attached to $\mathrm{C}_{60}$ with several methods [18-20]. CNT-Al $\mathrm{Al}_{13}$ cluster system was designed for molecular recognition [21], single-walled carbon nanotubes/iron oxide nanocomposite was used as imaging agents [22] and several kind of carbon nanotube-Au nanocomposites with new properties have also been prepared [23-26]. Like fullerene or carbon nanotubes-based composite, the combination of graphene and metal nanoparticles may result in some useful carbonaceous materials for in-demand applications in industry [8].

Graphene-metal composites are usually obtained by reduction of the corresponding metallic (e.g. $\mathrm{AuCl}_{4}^{-}, \mathrm{PdCl}_{4}{ }^{2-}$ 
or $\mathrm{PtCl}_{4}{ }^{2-}$ ) ions by ethylene glycol [8], $\mathrm{NaBH}_{4}$ [16] or hydrazine hydrate [9] in the presence of soluble graphene oxide sheets. It has been found that the electrical conductivity of graphene oxide (GO) could be improved obviously by chemical reduction [27]. Unfortunately, there are some limitations for this method: first, the strong reducing agents such as hydrazine has potential health hazard to person; second, it is difficult to control the in situ growth of nanoparticles and it is also difficult to obtain metal decorated GO because GO will be reduced as well during the reductive process; third, most of the composites would tend to form irreversible agglomerates due to the strong van der Waals force between the thin carbonic layers, which make an obstacle for further study and applications [28].

Herein, we explore a new approach for preparing water-soluble aAuNPs-GO nanocomposite through the interaction of amino groups on $\mathrm{Au}$ nanoparticles with a GO plane, as illustrated in Scheme 1. Compared with previous routes of fabrication, ours covers three quite unusual attributes: first, the free $\mathrm{NH}_{2}$ groups on aAuNPs can be further used; second, the final nanocomposite preserved the functional groups on GO, which endows the composite with solubility in a variety of solvents and feasibility for further chemical modification; and third, attaching aAuNPs to GO preserves the structure of both. The design of proper graphene-based nanocomposites and further study of their electronic and catalytic character will probably open up new avenues for applications.

\section{Experimental}

\subsection{Chemicals}

Except for 4-aminobenzenethiol and 1-hexanethiol (>96.0\%, TCI, Japan), all other reagent-grade chemicals were purchased from Sinopharm Chemical Reagent Co., Ltd. (SCRC), China, without further purification. Milli-Q water (18.20 $\mathrm{M} \Omega \mathrm{cm}$ resistivity) was used in the preparation procedure of GO and monolayer protected aAuNPs.

\subsection{Synthesis of aAuNPs $\left(0.045 \mathrm{mmol}\right.$ of $\mathrm{SHC}_{6} \mathrm{H}_{4} \mathrm{NH}_{2}$ and $0.045 \mathrm{mmol}$ of $\left.\mathrm{CH}_{3}\left(\mathrm{CH}_{2}\right)_{4} \mathrm{CH}_{2} \mathrm{SH}\right)$ and hAuNPs (0.09 mmol of $\left.\mathrm{CH}_{3}\left(\mathrm{CH}_{2}\right)_{4} \mathrm{CH}_{2} \mathrm{SH}\right)$}

Monolayer protected AuNPs were synthesized according to our previous work [29].

\subsection{Preparation of GO nanosheets}

GO was made according to a modified Hummers method [30]. In detail, about $20 \mathrm{~g}$ of graphite powder was put into an $80^{\circ} \mathrm{C}$ solution of concentrated $\mathrm{H}_{2} \mathrm{SO}_{4}(30 \mathrm{~mL}), \mathrm{K}_{2} \mathrm{~S}_{2} \mathrm{O}_{8}$ $(10 \mathrm{~g})$, and $\mathrm{P}_{2} \mathrm{O}_{5}(10 \mathrm{~g})$. After $6 \mathrm{~h}$, the resultant mixture was allowed to cool to room temperature, and then was carefully diluted with a large amount of Milli-Q water, filtered, and washed on the filter till the $\mathrm{pH}$ of rinsed water reached neutral. The product was dried in air. Then $20 \mathrm{~g}$ of the preoxidized graphite powder was put into $460 \mathrm{~mL}$ of cooled $\left(0^{\circ} \mathrm{C}\right)$ concentrated $\mathrm{H}_{2} \mathrm{SO}_{4}$. Subsequently, about $60 \mathrm{~g}$ $\mathrm{KMnO}_{4}$ was added gradually with stirring and cooling. The mixture was then stirred at $35^{\circ} \mathrm{C}$ for $2 \mathrm{~h}$. After that, ultrapure water $(920 \mathrm{~mL})$ was slowly added and the mixture was maintained for $15 \mathrm{~min}$. The reaction was terminated by addition of ultrapure water $(2800 \mathrm{~mL})$ and $50 \mathrm{~mL}, 30 \%$ $\mathrm{H}_{2} \mathrm{O}_{2}$ solution. The solid product was separated by filtering and washed with $5000 \mathrm{~mL}, 1: 10 \mathrm{HCl}$ solution. Then, GO product was suspended in Milli-Q water and sonicated for 2 h. The supernanant yellow-brown sol was subjected to dialysis membrane (MWCO 8000-15000) with ultrapure water to completely remove metal ions and acids followed by filtering and dried at room temperature in a vacuum, forming a shiny black and flexible paper-like material.

\subsection{Synthesis of aAuNPs-GO and hAuNPs-GO}

GO could be easily suspended in Dimethyl sulfoxide (DMSO) due to epoxy and hydroxyl groups [31]. In our experiment, $50 \mathrm{mg}$ of as-prepared GO paper-like material was dissolved in $50 \mathrm{~mL}$ of DMSO by sonication for $1 \mathrm{~h}$, forming a stable GO suspension. After that, $10 \mathrm{~mL}$ DMSO solution containing $20 \mathrm{mg}$ purified AuNPs (aAuNPs or hAuNPs) was added while stirring the mixture strongly. After addition of AuNPs, dark suspension of the mixture was continually stirred by a Teflon-coated magnetic bar for three days. For purification, the reaction mixture was centrifuged and the precipitation was washed three times with toluene. To completely remove the free AuNPs, the product was sonicated in Milli-Q water for $30 \mathrm{~min}$. Finally, the pure supernanant aAuNPs-GO or hAuNPs-GO nanocomposite solution was obtained through centrifuge followed by decantation. The fabrication procedure is outlined in Scheme 1.

\subsection{Characterization}

Fourier transform infrared spectroscopy (FTIR) spectra were collected on a Thermo Nicolet Avatar 370 FT-IR spectrophotometer at a resolution of $4 \mathrm{~cm}^{-1}$ and 32 scans in absorption mode. Investigation of the structure was conducted by transmission electron microscopy (TEM) using a JEOL 2010 TEM instrument with an acceleration voltage of $200 \mathrm{kV}$. X-ray photoelectron spectroscopy (XPS) results were recorded on a Multitechnique Electron Spectrometer for Surface Analysis (Kratos Axis Ultra DLD, UK) using monochromated $\mathrm{Al} \mathrm{K \alpha} 15 \mathrm{kV}, 10 \mathrm{~mA}$ as excitation source. Colloidal suspension of GO was deposited on freshly cleaved mica sheets for atomic force microscopy (AFM) imaging. AFM imaging of GO was performed in tapping mode on a Multimode Nanoscope III from Digital Instruments (Veeco Metrology Group, USA). Raman spectra were recorded on a LabRAM-1B micro spectrometer (France) with 

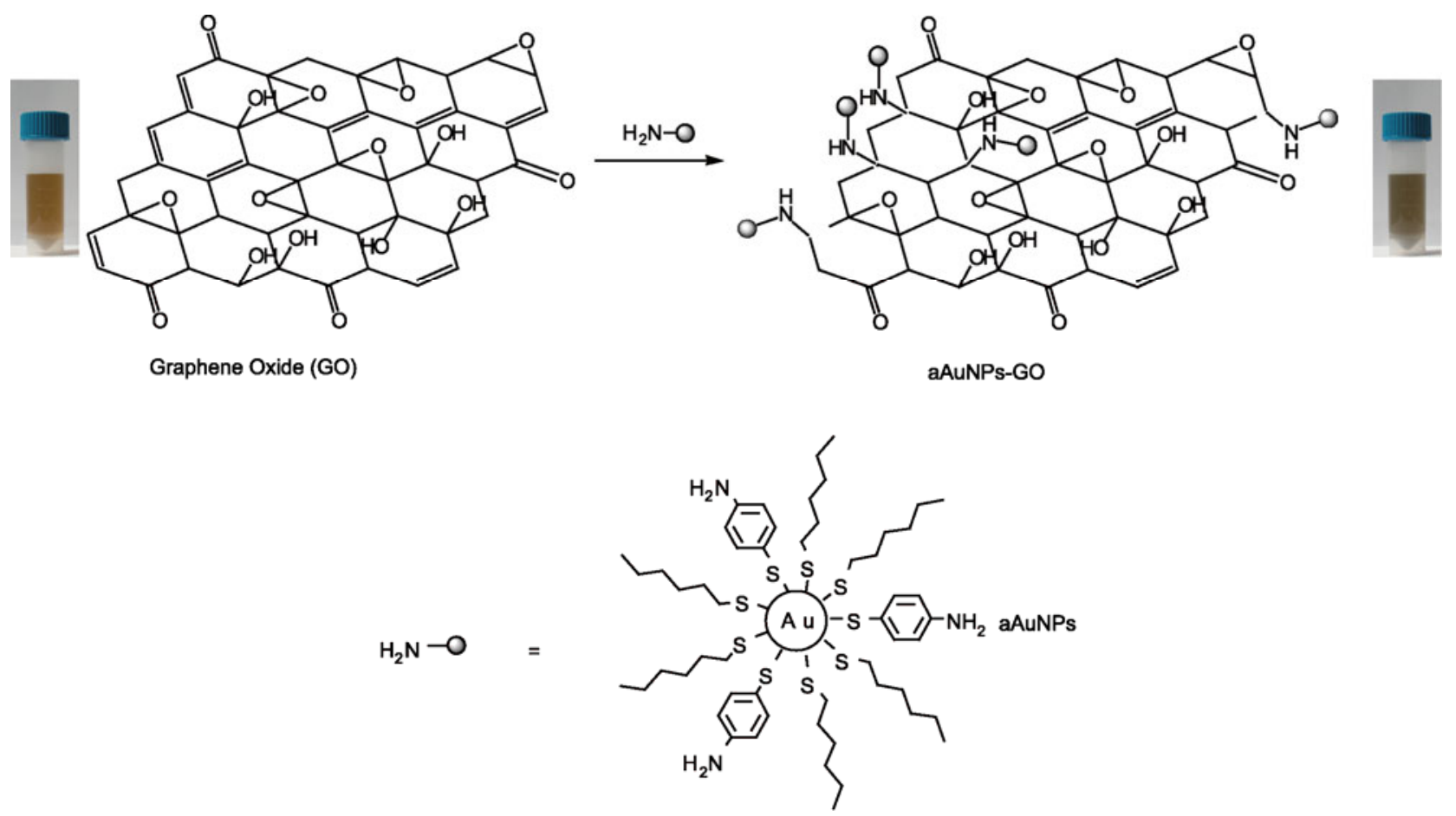

Scheme 1 Schematic illustration of the preparation procedure for aAuNPs-GO.

$632.8 \mathrm{~nm}$ laser excitation. Electrochemical impedance (EIS) experiments were carried out on a CHI $660 \mathrm{~b}$ electrochemical workstation (CH Instruments, Chenhua Co., Shanghai, China) by using a conventional three-electrode cell, including a modified glassy carbon electrode as working electrode, an $\mathrm{Ag} / \mathrm{AgCl}$ (saturated $\mathrm{KCl}$ ) electrode as reference electrode, and a platinum wire as counter electrode, which was measured in the presence of $5.0 \mathrm{mmol} / \mathrm{L} \quad \mathrm{K}_{3}\left[\mathrm{Fe}(\mathrm{CN})_{6}\right] / \mathrm{K}_{4}$ $\left[\mathrm{Fe}(\mathrm{CN})_{6}\right](1: 1)$ mixed solution as a redox probe in 0.1 $\mathrm{mol} / \mathrm{L} \mathrm{KCl}$. The AC voltage amplitude was $5 \mathrm{mV}$ and the voltage frequencies ranged from 0.1 to $10^{4} \mathrm{~Hz}$.

\section{Results and discussion}

Single GO layer could be easily formed due to its high dispersion stability in water and some polar reagents, which make it a promising candidate in applications such as photocatalysts [14], liquid crystal devices [32], and nanocomposites [8,27].

In the present study, we have succeeded in carrying out decoration of GO with aAuNPs in DMSO, which is illustrated in Scheme 1. It starts with dispersion of GO in DMSO, aAuNPs were then added and the mixture was allowed to stir for three days at room temperature so as to produce a dispersion of GO sheets linked with aAuNPs.

The morphology and structure of AuNPs and AuNPs-GO composites were demonstrated through transmission electron microscopy (TEM). The sizes of hAuNPs and aAuNPs (black dots) are in the range of 2-5 nm (Figure 1(a) and (c)).
TEM images also showed aggregation of hAuNPs on the surface of GO (Figure 1(b)). Their weak adhesion could be along with high mobility, although we do not thoroughly understand why hAuNPs aggregate on GO platelets. Similar findings have been previously reported on the surface of carbon nanotubes [26]. In contrast to hAuNPs, aAuNPs are mainly dispersed on the edge of GO sheets (Figure 1(d)). We suggest that a Michael addition-like reaction between $\mathrm{NH}_{2}$ groups, which may act as a nucleophile located on the surface of aAuNPs, and the double bonds of carbon plane results in less surface mobility of aAuNPs and causes less aggregation [30,31]. It is noteworthy that the size of aAuNPs is almost unchanged before attachment to the surface of GO.

Figure 2 shows atomic force microscopy (AFM) images of GO and aAuNPs-GO composite. Tapping-mode AFM was used to characterize GO (Figure 2(a)). From the height profile, we can see that GO nanosheet has an average thickness of $1 \mathrm{~nm}$, which demonstrates the presence of individual GO sheets in suspension [33]. And it is evident that individual aAuNPs are coupled to a single-layer GO edge that has plenty of carbonyl groups and conjugated double bonds [34]. The line of interest in Figure 2(b) reveals that aAuNPs-GO with height ca. $3.6 \mathrm{~nm}$ has been obtained in our contribution, which indicates that the initial binding of aAuNPs through addition reaction remains unaffected and thus is likely to keep the morphology undamaged in the composites.

X-ray photoelectron spectroscopy (XPS) was employed to further investigate the interaction between aAuNPs and 


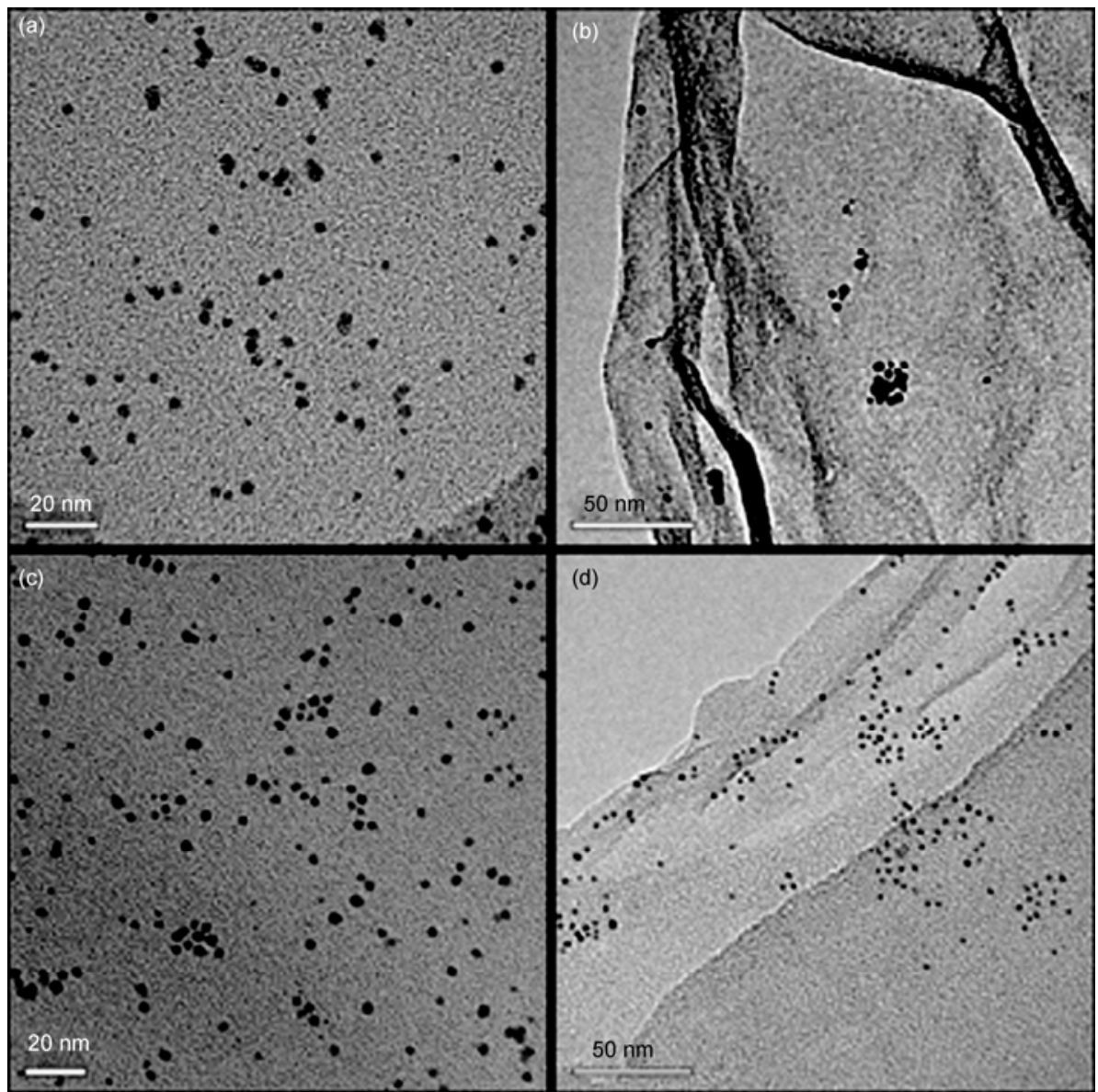

Figure 1 epresentative TEM images of (a) hAuNPs, (b) hAuNPs-GO, (c) aAuNPs, and (d) aAuNPs-GO.
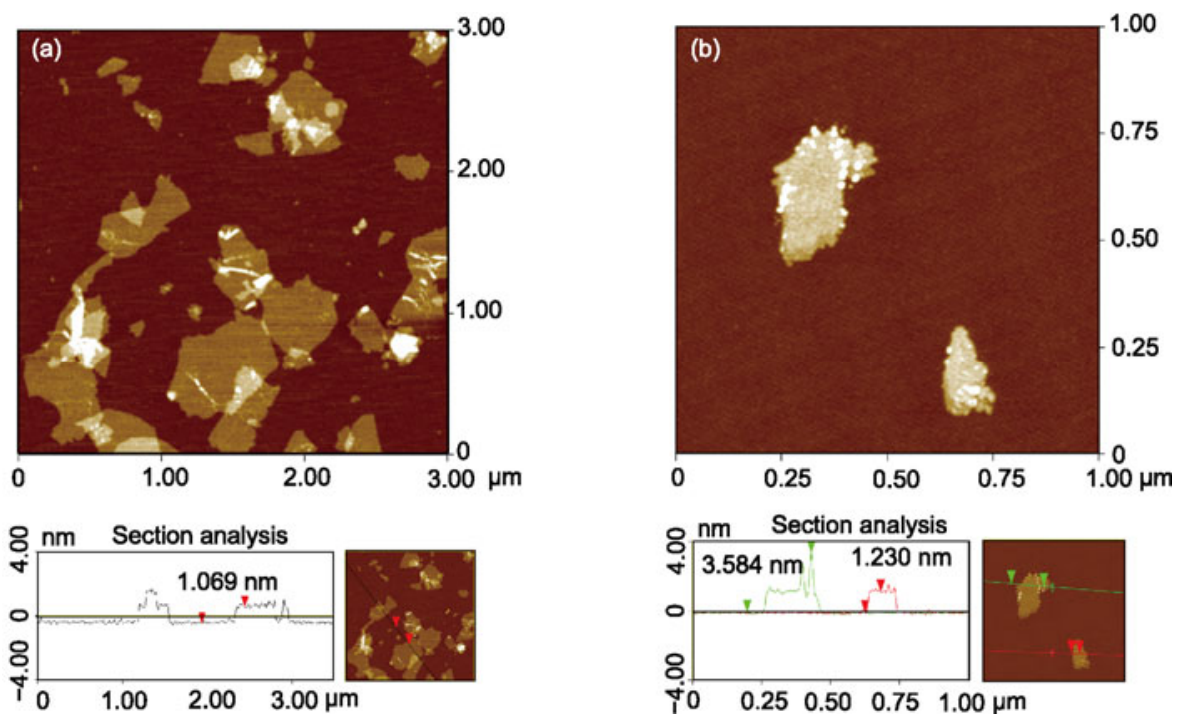

Figure 2 Tapping-mode AFM images and cross-sectional analysis of (a) GO and (b) aAuNPs-GO platelets dispersed on mica.

GO. Figure 3 shows the survey spectra of the samples and higher resolution peaks of $\mathrm{C} 1 \mathrm{~s}$ and $\mathrm{Au} 4 \mathrm{f}$ areas, respectively. The C 1s core-level spectra of GO shown in Figure 3(a) can be deconvoluted into three species at $284.8,286.8$, and $288.2 \mathrm{eV}$, respectively. Except for the characteristic gra- phitic carbon peak at $284.8 \mathrm{eV}$, the other two peaks are assigned to epoxy/ether group $(286.8 \mathrm{eV})$ and $\mathrm{C}=\mathrm{O}$ group $(288.2 \mathrm{eV})$ [35-37], respectively. After addition reaction, the peaks at 286.8 and $288.2 \mathrm{eV}$ decreased slightly relative to that at $284.8 \mathrm{eV}$, which suggested $\mathrm{C}=\mathrm{O}$ and epoxy/ether 

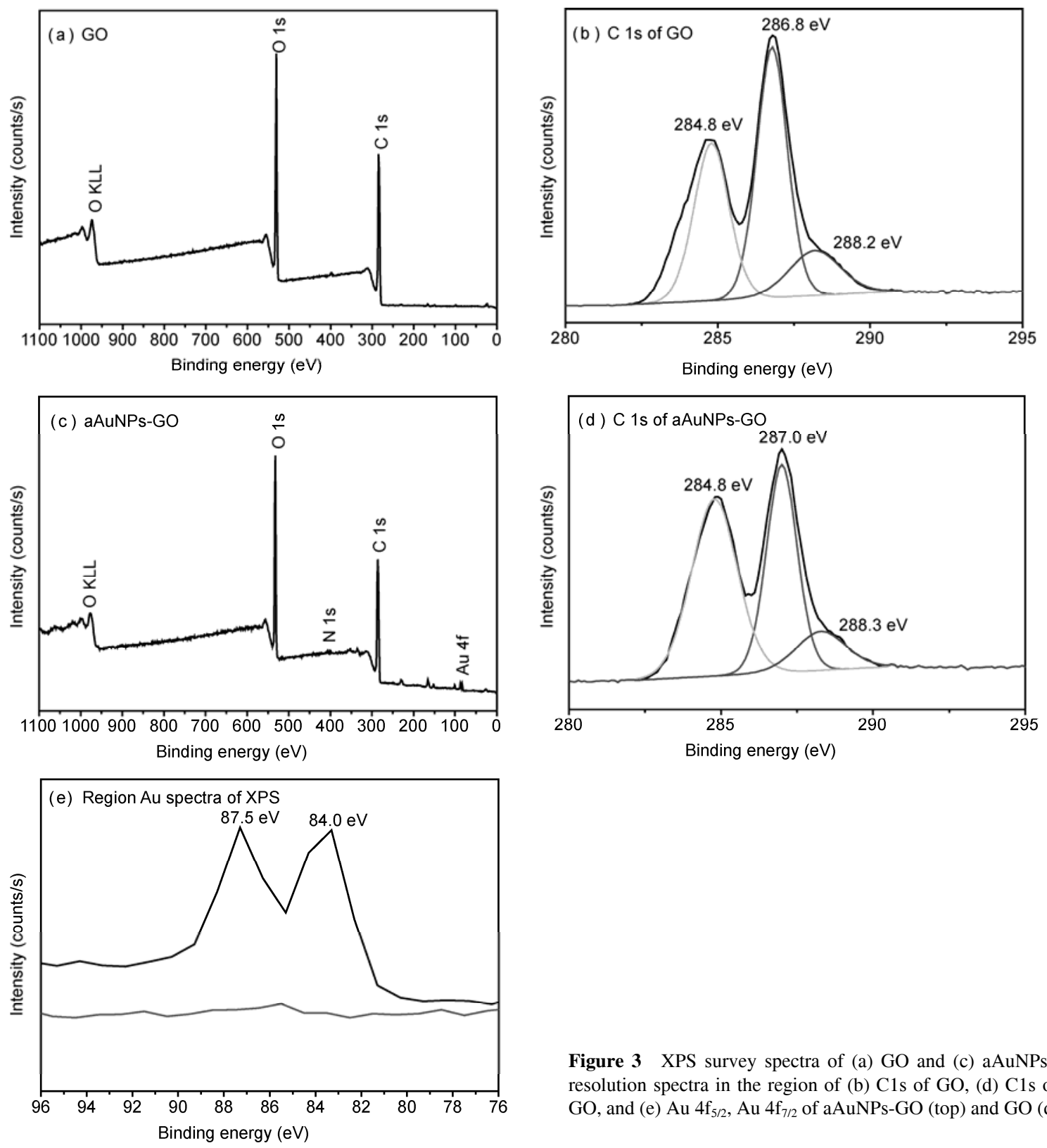

Figure 3 XPS survey spectra of (a) GO and (c) aAuNPs-GO; highresolution spectra in the region of (b) C1s of GO, (d) C1s of aAuNPs$\mathrm{GO}$, and (e) $\mathrm{Au} 4 \mathrm{f}_{5 / 2}, \mathrm{Au} 4 \mathrm{f}_{7 / 2}$ of aAuNPs-GO (top) and GO (down).

groups were changed little during the bonding process. This indicates that in this case, aAuNPs were homogeneously coalesced onto GO plane mainly through the mechanism of a Michael addition-like reaction without affecting other functional groups, except for $\mathrm{C}=\mathrm{C}$ bonds [38]. Figure 3(e) further confirms the presence of $\mathrm{Au}$ in the aAuNPs-GO composite. However, $\mathrm{Au}$ is absent in GO film. The data here is consistent with the observations of TEM and AFM, and again indicates the addition reaction.

For $\mathrm{sp}^{2}$ carbon nanomaterials such as graphene, useful information about crystallite size, clustering of the $\mathrm{sp}^{2}$ phase, the presence of $\mathrm{sp}^{2}-\mathrm{sp}^{3}$ hybridization and the introduction of chemical impurities can be offered by Raman spectroscopy [39]. Raman spectra further prove the structural change

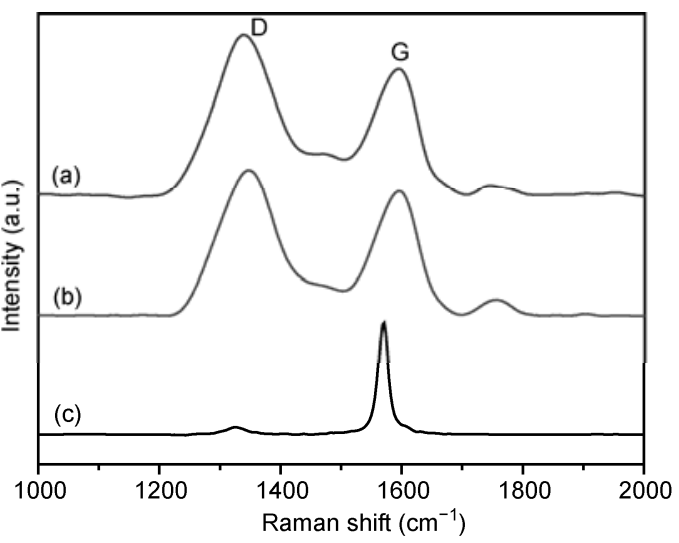

Figure 4 Raman spectra of (a) aAuNPs-GO, (b) GO, and (c) graphite. 
before and after addition reaction. In all samples, two main characteristic peaks are clearly observed (Figure 4), corresponding to the D band and $\mathrm{G}$ band at 1326 and $1571 \mathrm{~cm}^{-1}$, respectively. The $\mathrm{D}$ and $\mathrm{G}$ band intensity ratio shows the carbon ratio of $\mathrm{sp}^{2} / \mathrm{sp}^{3}$, which is a measure of the extent of disorder [40]. In our experiments, the D/G ratios of graphite, GO sheets, and aAuNPs-GO are respectively 0.06, 1.17 and 1.27 , reflecting the increase in structural disorder, which provided more evidence for the occurrence of addition reaction.

Further evidence of covalent bonding between aAuNPs and GO sheets is revealed by Fourier transform infrared (FTIR) spectra shown in Figure 5. The spectrum of GO is in good agreement with previous work [31]. The most characteristic features of $\mathrm{GO}$ are $\mathrm{O}-\mathrm{H}$ stretching vibrations at $3397 \mathrm{~cm}^{-1}, \mathrm{C}=\mathrm{O}$ stretching vibrations from carbonyl groups at $1732 \mathrm{~cm}^{-1}$, carbon skeletal vibrations at $1628 \mathrm{~cm}^{-1}$ and $\mathrm{C}-\mathrm{O}$ stretching vibrations at $1079 \mathrm{~cm}^{-1}$ in Figure 5(c). FTIR spectrum of aAuNPs-GO differs from that of GO as proved by the dramatic weakening of the peak at $1628 \mathrm{~cm}^{-1}$. It suggests that the addition reaction occupied some conjugated double bonds situated on the edge of GO so that the $\mathrm{C}=\mathrm{C}$ peak became weaker compared with $\mathrm{GO}$, which is consistent with TEM and AFM results. The peak at 2922 $\mathrm{cm}^{-1}$ in aAuNPs-GO arises from $\mathrm{CH}_{2}$ groups of alkyl chains assigning to aAuNPs. Especially, a band at $1318 \mathrm{~cm}^{-1}$ assigned to $\mathrm{C}-\mathrm{N}$ stretching vibrations $\left(v_{\mathrm{C}-\mathrm{N}}\right.$ binding with aromatic ring structure) of 4-aminobenzenethiol on $\mathrm{Au}$ [41]. These observations indicate that aAuNPs were successfully introduced onto GO through covalent bonding. Furthermore, the residual free $\mathrm{NH}_{2}$ groups on aAuNPs can be used for other molecular modifications.

Electrochemical impedance spectroscopy (EIS) is the most straight-forward and crucial technique to reflect the changes of electrical conductivity of an electrode/electrolyte interface [42]. The good conducting feature of aAuNPs-GO nanocomposite was obtained through EIS as shown in Figure 6. The Nyquist plot of EIS measurement presents a semicircle correlated with charge transfer resistance and a straight slopping line ascribed to mass transfer. It is observed

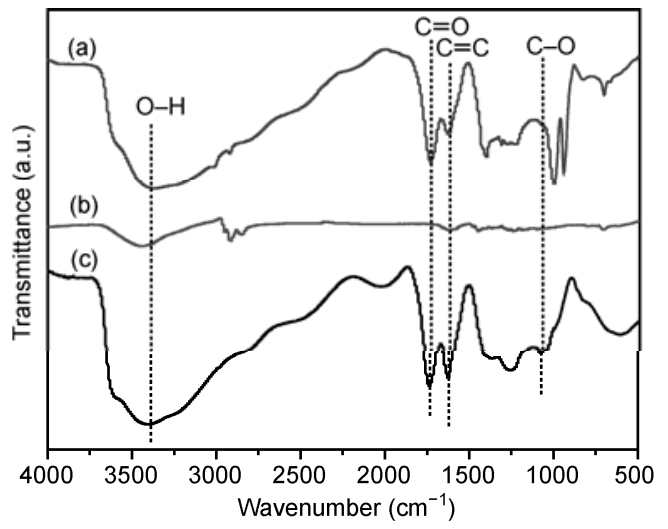

Figure 5 FTIR spectra of (a) aAuNPs-GO, (b) aAuNPs, and (c) GO.

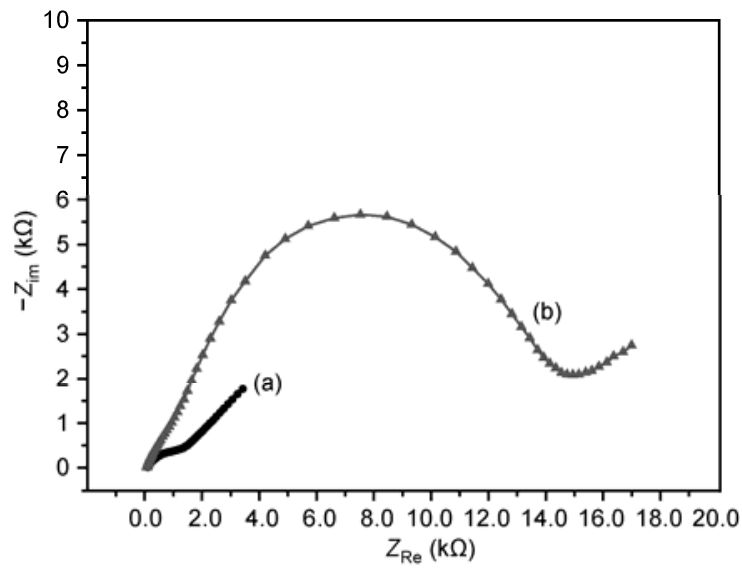

Figure 6 Nyquist plot for the electrochemical impedance measurements in the presence of $5.0 \mathrm{mmol} / \mathrm{L} \mathrm{K}_{3}\left[\mathrm{Fe}(\mathrm{CN})_{6}\right] / \mathrm{K}_{4}\left[\mathrm{Fe}(\mathrm{CN})_{6}\right](1: 1)$ as redox probe and $0.1 \mathrm{~mol} / \mathrm{L} \mathrm{KCl}$ as supporting electrolyte: (a) aAuNPs-GO and (b) GO modified electrodes.

that with introduction of aAuNPs the semicircle in the plot was shortened, which indicates that the charge transfer resistance on the interface and solid-state interface layer resistance were decreased [43]. The additive of Au particles is responsible for the increase in electronic conductivity of aAuNPs-GO composite, though we do not know the detail mechanism. The improved conductivity of aAuNPs-GO nanocomposite would be beneficial to its electrocatalytic application.

\section{Conclusions}

In summary, we have developed a facile and useful chemical approach to prepare aAuNPs-GO nanocomposites. In our procedure, aAuNPs formed first and then were introduced onto the two-dimensional planar structure, which is beneficial for the following utilization of residual $\mathrm{NH}_{2}$ groups on aAuNPs. Furthermore, the binding of the particles retains good solubility of GO sheets. With further optimization of various parameters, such as the nanoparticle type in the composite and the change in number of $\mathrm{NH}_{2}$ groups, aAuNPs-GO is expected to become a universal platform for graphene-based nanocomposite research. In addition, the high density of the Au core in the composite could provide good contrast of the metal on the GO surface. In the future, this will facilitate the recognition of GO in a cellular environment. Therefore, our synthesized nanocomposites will also have potential applications as labeling agents.

This work was supported by the National Natural Science Foundation of China (20725516, 90913014, 10975179), the National Basic Research Program of China (2007CB936000), Ministry of Health (2009ZX10004301) and Shanghai Municipal Commission for Science and Technology (0952nm04600). 
1 Castro Neto A H. The carbon new age. Mater Today, 2010, 13: 1217

2 Geim A K, Novoselov K S. The rise of graphene. Nat Mater, 2007, 6: 183-191

3 Katsnelson M I. Graphene: Arbon in two dimensions. Mater Today, 2007, 10: 20-27

4 Novoselov K S, Geim A K, Morozov S V, et al. Electric field effect in atomically thin carbon films. Science, 2004, 306: 666-669

5 Novoselov K S, Geim A K, Morozov S V, et al. Two-dimensional gas of massless dirac fermions in graphene. Nature, 2005, 438: 197200

6 Zhang Y, Small J P, Amori M E S, et al. Electric field modulation of galvanomagnetic properties of mesoscopic graphite. Phys Rev Lett, 2005, 94: 176803

7 Berger C, Song Z, Li T, et al. Ultrathin epitaxial graphite: $2 \mathrm{~d}$ electron gas properties and a route toward graphene-based nanoelectronics. J Phys Chem B, 2004, 108: 19912-19916

8 Xu C, Wang X, Zhu J. Graphene-metal particle nanocomposites. J Phys Chem C, 2008, 112: 19841-19845

9 Hassan H M A, Abdelsayed V, Khder A E R S, et al. Microwave synthesis of graphene sheets supporting metal nanocrystals in aqueous and organic media. J Mater Chem, 2009, 19: 3832-3837

10 Fissan H, Kennedy M K, Krinke T J, et al. Nanoparticles from the gas phase as building blocks for electrical devices. J Nanopart Res, 2003, 5: 299-310

11 Gao W, Alemany L B, Ci L, et al. New insights into the structure and reduction of graphite oxide. Nat Chem, 2009, 1: 403-408

12 Meyer J C, Geim A K, Katsnelson M I, et al. The structure of suspended graphene sheets. Nature, 2007, 446: 60-63

13 Subrahmanyam K S, Vivekchand S R C, Govindaraj A, et al. A study of graphenes prepared by different methods: Characterization, properties and solubilization. J Mater Chem, 2008, 18: 1517-1523

14 Zhang H, Lü X, Li Y, et al. P25-graphene composite as a high performance photocatalyst. ACS Nano, 2009, 4: 380-386

15 Williams G, Seger B, Kamat P V. $\mathrm{TiO}_{2}$-graphene nanocomposites. $\mathrm{UV}$-assisted photocatalytic reduction of graphene oxide. ACS Nano, 2008, 2: 1487-1491

16 Muszynski R, Seger B, Kamat P V. Decorating graphene sheets with gold nanoparticles. J Phys Chem C, 2008, 112: 5263-5266

17 Lu G, Mao S, Park S, et al. Facile, noncovalent decoration of graphene oxide sheets with nanocrystals. Nano Res, 2009, 2: 192-200

18 Shon Y S, Choo H. [60]Fullerene-linked gold nanoparticles: Synthesis and layer-by-layer growth on a solid surface. Chem Commun, 2002, 21: 2560-2561

19 Sudeep P K, Ipe B I, Thomas K G, et al. Fullerene-functionalized gold nanoparticles. A self-assembled photoactive antenna-metal nanocore assembly. Nano Lett, 2001, 2: 29-35

20 Lu F, Xiao S, Li Y, et al. Fullerene-functionalized gold core-shell nanoparticles: Preparation and optical limiting properties. Inorg Chem Commun, 2004, 7: 960-962

21 Zhao Q, Buongiorno Nardelli M, Lu W, et al. Carbon nanotube-metal cluster composites: A new road to chemical sensors. Nano Lett, 2005, 5: $847-851$

22 Choi J H, Nguyen F T, Barone P W, et al. Multimodal biomedical imaging with asymmetric single-walled carbon nanotube/iron oxide nanoparticle complexes. Nano Lett, 2007, 7: 861-867

23 Guo L, Peng Z. One-pot synthesis of carbon nanotube-polyanilinegold nanoparticle and carbon nanotube-gold nanoparticle composites by using aromatic amine chemistry. Langmuir, 2008, 24: 8971-8975

24 Wang Z, Li M, Zhang Y, et al. Thionine-interlinked multi-walled carbon nanotube/gold nanoparticle composites. Carbon, 2007, 45: 2111-2115

25 Raghuveer M S, Agrawal S, Bishop N, et al. Microwave-assisted single-step functionalization and in situ derivatization of carbon nanotubes with gold nanoparticles. Chem Mater, 2006, 18: 1390-1393

26 Herrero M A, Guerra J, Myers V S, et al. Gold dendrimer encapsulated nanoparticles as labeling agents for multiwalled carbon nanotubes. ACS Nano, 2010, 4: 905-912

27 Stankovich S, Dikin D A, Dommett G H B, et al. Graphene-based composite materials. Nature, 2006, 442: 282-286

28 Liu J B, Fu S H, Yuan B, et al. Toward a universal "adhesive nanosheet" for the assembly of multiple nanoparticles based on a protein-induced reduction/decoration of graphene oxide. J Am Chem Soc, 2010, 132: 7279

29 Geng M, Zhang Y, Huang Q, et al. Functionalization of C-60 with gold nanoparticles. Carbon, 2010, 48: 3570-3574

30 Kovtyukhova N I, Ollivier P J, Martin B R, et al. Layer-by-layer assembly of ultrathin composite films from micron-sized graphite oxide sheets and polycations. Chem Mater, 1999, 11: 771-778

31 Paredes J I, Villar-Rodil S, Martínez-Alonso A, et al. Graphene oxide dispersions in organic solvents. Langmuir, 2008, 24: 10560-10564

32 Blake P, Brimicombe P D, Nair R R, et al. Graphene-based liquid crystal device. Nano Lett, 2008, 8: 1704-1708

33 Stankovich S, Dikin D A, Piner R D, et al. Synthesis of graphenebased nanosheets via chemical reduction of exfoliated graphite oxide. Carbon, 2007, 45: 1558-1565

34 Lee D W, De Los Santos V L, Seo J W, et al. The structure of graphite oxide: Investigation of its surface chemical groups. J Phys Chem B, 2010, 114: 5723-5728

35 Park S, An J, Piner R D, et al. Aqueous suspension and characterization of chemically modified graphene sheets. Chem Mater, 2008, 20: 6592-6594

36 Yang H, Li F, Shan C, et al. Covalent functionalization of chemically converted graphene sheets via silane and its reinforcement. J Mater Chem, 2009, 19: 4632-4638

37 Yang H, Shan C, Li F, et al. Covalent functionalization of polydisperse chemically-converted graphene sheets with amine-terminated ionic liquid. Chem Commun, 2009, 26: 3880-3882

38 Mather B D, Viswanathan K, Miller K M, et al. Michael addition reactions in macromolecular design for emerging technologies. Prog Polym Sci, 2006, 31: 487-531

39 Dresselhaus M S, Jorio A, Hofmann M, et al. Perspectives on carbon nanotubes and graphene raman spectroscopy. Nano Lett, 2010, 10: 751-758

40 Fang M, Wang K, Lu H, et al. Covalent polymer functionalization of graphene nanosheets and mechanical properties of composites. J Mater Chem, 2009, 19: 7098-7105

41 Shan C, Yang H, Han D, et al. Water-soluble graphene covalently functionalized by biocompatible poly-1-lysine. Langmuir, 2009, 25: 12030-12033

42 Zhu C, Guo S, Fang Y, et al. Reducing sugar: New functional molecules for the green synthesis of graphene nanosheets. ACS Nano, 2010, 4: 2429-2437

43 He B L, Dong B, Li H L. Preparation and electrochemical properties of Ag-modified $\mathrm{TiO}_{2}$ nanotube anode material for lithium-ion battery. Electrochem Commun, 2007, 9: 425-430

Open Access This article is distributed under the terms of the Creative Commons Attribution License which permits any use, distribution, and reproduction in any medium, provided the original author(s) and source are credited. 